\title{
Do dissociated or associated phoria predict the comfortable prism?
}

\author{
Joanna M. N. Otto • Miriam Kromeier • Michael Bach • \\ Guntram Kommerell
}

Received: 18 June 2007 /Revised: 21 January 2008 /Accepted: 19 February 2008 / Published online: 1 April 2008

(C) Springer-Verlag 2008

\begin{abstract}
Background Dissociated and associated phoria are measures of latent strabismus under artificial viewing conditions. We examined to what extent dissociated and associated phoria predict the "comfortable prism", i.e. the prism that appears most comfortable under natural viewing conditions.

Methods For associated phoria, a configuration resembling the Mallett test was employed: both eyes were presented with a fixation cross, surrounded by fusionable objects. Nonius lines served as monocular markers. For dissociated phoria, the left eye was presented with all the Mallett elements, while only a white spot was presented to the right eye. To determine the comfortable prism, all the Mallett elements, including the Nonius lines, were shown to both eyes. In each of the three tests, the observer had to adjust a pair of counterrotating prisms. To avoid any (possibly prejudiced) influence of the experimenter, the prismatic power was recorded with a potentiometer. Twenty nonstrabismic subjects with a visual acuity of $\geq 1.0$ in each eye were examined.

Results The range of the intertrial mean was for dissociated phoria from +9.3 eso to $-5.9 \mathrm{~cm} / \mathrm{m}$ exo, for associated phoria from +11.2 eso to $-3.3 \mathrm{~cm} / \mathrm{m}$ exo, and for the comfortable prism from +4.8 eso to $-4.1 \mathrm{~cm} / \mathrm{m}$ exo $(\mathrm{cm} / \mathrm{m}=$ prism dioptre). In most observers, the phoria parameters differed greatly from the comfortable prism. On average, the phoria values were shifted about $2 \mathrm{~cm} / \mathrm{m}$ towards the eso direction in relation to the comfortable prism (associated phoria not less than dissociated phoria).
\end{abstract}

J. M. N. Otto $\cdot$ M. Kromeier • M. Bach • G. Kommerell $(\bowtie)$

Universitäts-Augenklinik,

Killianstr. 5,

79106 Freiburg, Germany

e-mail: guntram.kommerell@uniklinik-freiburg.de
Conclusions The deviation of both, dissociated and associated phoria, from the comfortable prism suggests that the abnormal viewing conditions under which the phoria parameters are determined induce artefacts. Accordingly, the findings cast doubt on current textbook recommendations to use dissociated or associated phoria as a basis for therapeutic prisms. Rather, patients should be allowed to determine their comfortable prism under natural viewing conditions.

Keywords Heterophoria $\cdot$ Fixation disparity $\cdot$ Mallett unit . Aligning prism $\cdot$ Asthenopia

\section{Introduction}

Since Ogle's work [14] dissociated phoria has been distinguished from associated phoria. Dissociated phoria is defined as a deviation from the orthovergence position that occurs when no fusionable contours are provided. Associated phoria is a deviation of the eyes that appears under prism correction of fixation disparity: associated phoria equals the "aligning prism" [1] that nullifies fixation disparity. To avoid the technical requirements for measuring the eye position objectively, fixation disparity is commonly determined according to the observer's directional perception of monocular Nonius lines, embedded in binocular contours. Both dissociated [11, 18] and associated phoria $[9,19]$ have been recommended as indicators for prismatic correction in the case of asthenopia, but recently associated phoria has been preferred, because the stimulus for associated phoria appears to be more natural, in that both eyes are presented with a nearly identical configuration $[1$, 4, 5, 16, 20]. Moreover, Yekta et al. [20] found the correlation of asthenopic complaints to be significant only with associated, not with dissociated phoria. 
Kromeier et al. [8], however, suggested that dissociated and associated phoria should be similar, provided the accommodative demand in the two procedures is equal. Their argument was based on the consideration that the feedback loop for fusion is opened under both conditions. Concerning dissociated phoria, it is obvious that the feedback loop cannot operate; fusionable contours are absent, hence there is no error signal. Concerning associated phoria, the argument is more complicated because of the fundamental difference between fixation disparity and associated phoria. When the observer looks at a test for fixation disparity, any tendency to deviate from orthoposition is largely kept in check, because the disparity of the binocular contours provides an error signal: the fusional feedback loop is functioning. In the test for associated phoria, however, prisms are added. Prompt and repeated adjustment of the prismatic power nullifies any disparity of fusionable contours. This continuous and artificial resetting of the error signal to zero means that the fusional feedback loop cannot fulfill its purpose, namely stabilisation of a certain vergence angle.

The experimental results of Kromeier et al. supported their theoretical consideration. These authors suggested that the discrepancies between dissociated and associated phoria encountered in previous studies $[15,20]$ might be due to different accommodative stimuli.

To clarify this issue, we compared dissociated and associated phoria using the same object for fixation, thus keeping the accommodative demand similar. We further investigated to what extent dissociated and/or associated phoria predict the "comfortable prism". This term defines the prismatic power reached by the observers when they looked at a fully fusionable display, and tuned a pair of counterrotating prisms, called Herschel's or Risley's rotary prisms [17], such that the viewing of a fully fusionable object appeared most comfortable. Hence, the comfortable prism anticipated the real-life situation for which prismatic spectacles might be considered.

\section{Methods}

\section{Apparatus}

Stimuli were generated by a PowerMacintosh G4 and presented at a distance of $4.0 \mathrm{~m}$ on a 19" Philips GD403 CRT monitor with a resolution of $800 \times 600$ pixels. By means of liquid crystal shutter goggles (ELSA 3D Revelator), separate images were presented to the two eyes. The goggles were synchronized to the CRT refresh such that the frames were alternately presented to the right and left eyes. The refresh rate was $120 \mathrm{~Hz}$, i.e. $60 \mathrm{~Hz}$ for each eye, just above the flicker fusion frequency. The phosphor persistence of the monitor, measured with a photoelectric cell, was down to $10 \%$ after $4.0 \mathrm{~ms}$, i.e. within a shorter time than the frame time of $8 \mathrm{~ms}$. An alternate cover test assured us that this technique provided a complete separation between the images of the two eyes. Herschel's counterrotating prisms, manufactured by Zeiss, were mounted in front of their right eye (Fig. 1).

These prisms, equipped with a lever arm, allowed a variation of the prismatic power between $30 \mathrm{~cm} / \mathrm{m}$ base in and $30 \mathrm{~cm} / \mathrm{m}$ base out. A calibration performed with a laser beam showed that the readings on the scale of the prisms varied only about $2 \%$ around the true inflection. A cogwheel belt connected the prisms with a potentiometer that provided a signal linearly proportional to the prism power. The electric signal was directly transmitted to a computer.

During the trials the room was dark; in the intervals the room was moderately illuminated so that the test person was able to see a coloured print (Henri Matisse "Fleurs et céramique", $35 \times 40 \mathrm{~cm}$, mean luminance $13.8 \mathrm{~cd} / \mathrm{m}^{2}$ ) mounted directly above the monitor.

Test figures

\section{Dissociated phoria}

As depicted in Fig. 2a, the left eye was presented with a fixation cross, flanked by two O letters ("O X O"). Each of the three letters had a diameter of 10 arcmin and a line thickness of 1.5 arcmin. Above and below the cross were two Nonius lines, 20 arcmin high and 5 arcmin wide. The interval between the lines and the centre of the "O X O"

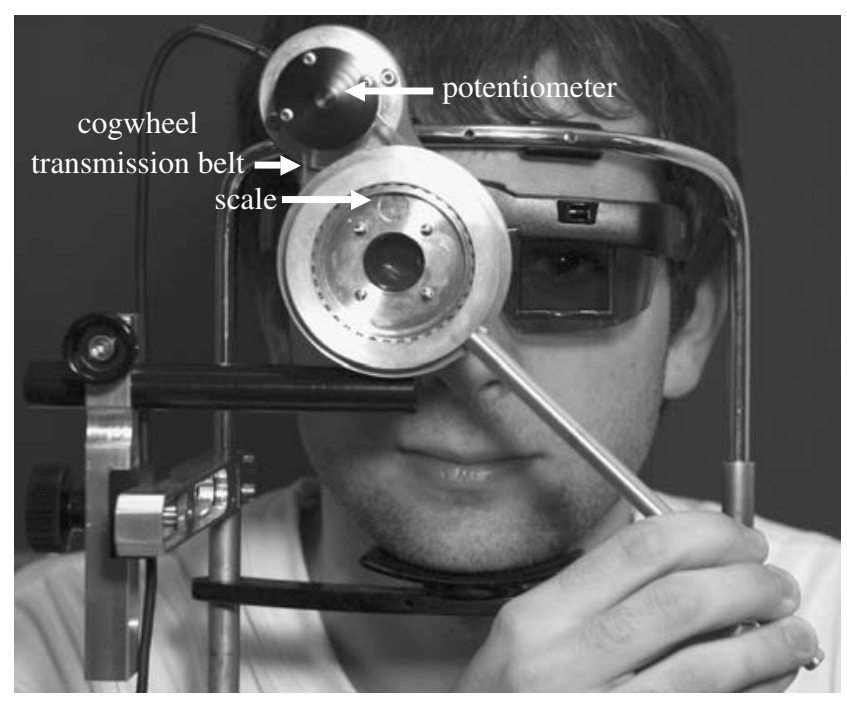

Fig. 1 Herschel's prisms mounted in front of the right eye. The observer, looking through shutter goggles, adjusts the prisms by means of a lever arm. A cogwheel belt transmits the prismatic power to a potentiometer 


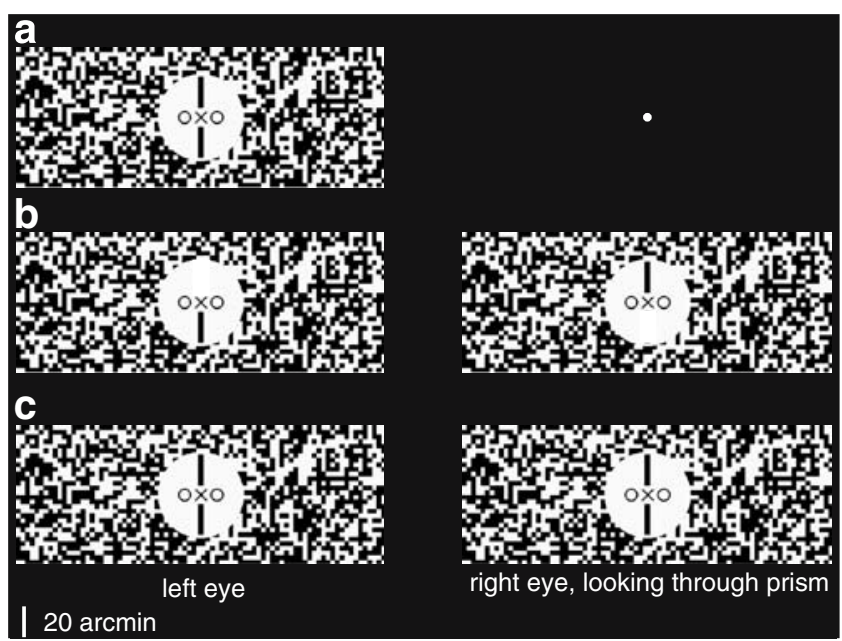

Fig. 2 Test figures. a Test for dissociated phoria. All the elements contained in the Mallett figure, including the two Nonius lines, are displayed to the left eye. The right eye is presented with a white dot on a homogenous dark field. b Test for associated phoria, according to Mallett [9]. c Test for the comfortable prism. An identical figure is displayed to both eyes

was 10 arcmin. A bright circular background of 40 arcmin radius, embedded in a random dot pattern, surrounded these dark features. The right eye was presented with a bright dot of 4.3 arcmin diameter on a homogenous dark field. The luminance of the dark features was $0.2 \mathrm{~cd} / \mathrm{m}^{2}$, and that of the bright features $22.6 \mathrm{~cd} / \mathrm{m}^{2}$ (measured through the liquid crystal shutter goggles). The luminance outside the monitor was $0.1 \mathrm{~cd} / \mathrm{m}^{2}$.

\section{Associated phoria}

As depicted in Fig. 2b, the configuration was similar to Mallett's test for fixation disparity [9]. The "O X O" served as a fusional stimulus. Vertical Nonius lines were used as monocular markers, in that the line above the fixation cross was shown to the right eye, and the line below the fixation cross to the left eye.

\section{Comfortable prism}

Both eyes were presented with all the Mallett elements including both Nonius lines (Fig. 2c).

\section{Procedure and instructions}

The observers were seated in a comfortable chair. During the trials, the observers had to rest their chin on a support, to lean their forehead against a bar, and to adjust Herschel's prisms with the lever arm. Before each trial, the experimenter set the prisms to \pm 0 . To limit prism adaptation [12], the time for the adjustment was restricted to 30 seconds. In the interval between the single trials (about 60 to $120 \mathrm{~s}$ ), the observers were encouraged to move their head freely and to look around in the room or to behold the Matisse painting.

Each of the three conditions (dissociated phoria, associated phoria, comfortable prism) was tested in a block of eight trials. The conditions were presented in the following order:

(1) Associated phoria. If the observers saw an offset between the Nonius lines, they had to align them with the least possible excursion of the lever arm so that they appeared as stable to each other as possible. This instruction resembles the suggestion of Karania and Evens [6] to observe whether one or both of the Nonius lines ever move. If an observer did not see an offset at the start of the trial, or the Nonius lines oscillated around zero, he or she was encouraged to "play" a little with the lever arm and set Herschel's prisms such that the Nonius lines became aligned as stably as possible. The observers were repeatedly reminded to look at the centre of the "O X O", not at the Nonius lines. Further, the observers were instructed to ignore if one Nonius line, or even if both of them seemed to disappear every now and then (fading). Most observers had such an experience. They had to adjust Herschel's prisms according to the percept when they saw both Nonius lines.

(2) Dissociated phoria. The observers were asked to align the white dot with the midline of the "O X O", neglecting any vertical offset of the white dot. While doing so, they were urged to strictly look at the centre of the "O X O", not at the white dot.

(3) Comfortable prism. The observers were asked to look at the centre of the "O X O" and to set the prisms such that viewing appeared most relaxing. They were encouraged to start with rather bold searching excursions and to refine the tuning gradually during the available 30 seconds.

To get used to the manoeuvring of Herschel's prisms, the experiment was preceded by a few trials in which the observers practised bringing the white dot to the midline of the "O X O" (test for dissociated phoria) and aligning the Nonius lines to each other (test for associated phoria).

To examine the reproducibility, we repeated the whole experiment for all observers in a second session after an interval of 24 to 130 days.

\section{Observers}

Twenty observers participated in the study (members of our department or recruits via a public advertisement, aged between 20 and 71 years, median 26.5 years). The observers were selected according to the following 4 
criteria: (1) Visual acuity of each eye (with full spherical and cylindrical correction) at least $1.0_{\text {decimal }}\left(=6 / 6_{\text {Snellen }}\right)$, (2) absence of strabismus, ascertained with the unilateral cover test, (3) absence of a prism in the spectacles, and (4) presence of random dot stereopsis (Lang Test 1). The observers were refracted without dilating their pupils, using streak retinoscopy and crossed cylinders, taking particular care to avoid any uncorrected hyperopia. During the experiment, the observers wore full spherical and cylindrical spectacle corrections. Care was taken to ensure that the glasses remained centred throughout the study. We did not inquire whether the observers had asthenopic symptoms or not.

We explained to the observers that the study intended to optimise the comfort of seeing. Otherwise, the observers were kept naive as to the purpose of the study. Each observer provided informed written consent to participate in the experiments. The study followed the tenets of the Declaration of Helsinki and was approved by the institutional human review board.

\section{Data acquisition and analysis}

The prismatic power derived from the potentiometer attached to Herschel's prisms was recorded by PowerLab with a sampling rate of $100 \mathrm{~Hz}$. To enable analysis of the dynamic behaviour of the adjustments we recorded the full 30 seconds of each trial. Offline examination was accomplished with Igor Pro ${ }^{\circledR}$ (Wavemetrics, Inc., Lake Oswego, OR, USA) and Statview ${ }^{\circledR}$ (Abacus Concepts, Inc., Berkeley, California, USA). Statistical analysis was performed with Statview $^{\circledR}$ and SPSS ${ }^{\circledR}$ (SPSS Inc., Chicago, Illinois, USA). We used multifactorial ANOVA (including condition, session and trial) and paired comparisons. For post-hoc tests, Bonferroni adjustment was performed.

\section{Results}

Inspection of curves

The curves obtained from the 20 observers varied considerably between the 8 trials. For example see Fig. 3. There was no trend, e.g. with the early trials (thin lines) yielding smaller values than the late ones (progressively thicker lines), or vice versa $(p=0.34)$. Most observers adjusted Herschel's prisms stepwise, reaching the final value after about 20 seconds. Therefore, we limited the numerical evaluation to the remaining 10 seconds.

For dissociated phoria, most observers saw an offset right at the beginning of each trial and promptly moved the lever arm accordingly.

For associated phoria, some observers initially did not see an offset between the Nonius lines. In this case, the observers followed the instruction to "play" a little with the lever arm and find a position in which the lines appeared as stable as possible.

For the comfortable prism, most observers started with rather bold searching excursions and refined the tuning gradually (Fig. 3, right panels). Only exceptionally, an observer ended up with a rather large range for the comfortable prism.

\section{Statistical analysis}

We averaged the 1000 values recorded during the last 10 seconds in each of the eight trials, as most observers reached the final value after about 20 seconds. As stated above, there was no trend between the eight trials, e.g. with the early trials yielding smaller values than the late ones, or vice versa $(p=0.34)$. This allowed us to treat the 8 trials together and calculate an intertrial mean \pm SEM (standard
Fig. 3 Adjustment of Herschel's prisms by observer \#10 for dissociated phoria, associated phoria, and the comfortable prism. First session. Positive values indicate base out prisms (eso deviation), negative values base in prisms (exo deviation). The upper panels show the original recordings of the eight single trials. The lower panels show the mean values (solid lines) with the standard error of the mean (SEM, dashed lines). Note that the scale of the ordinate for the comfortable prism differs from those in the two phoria conditions

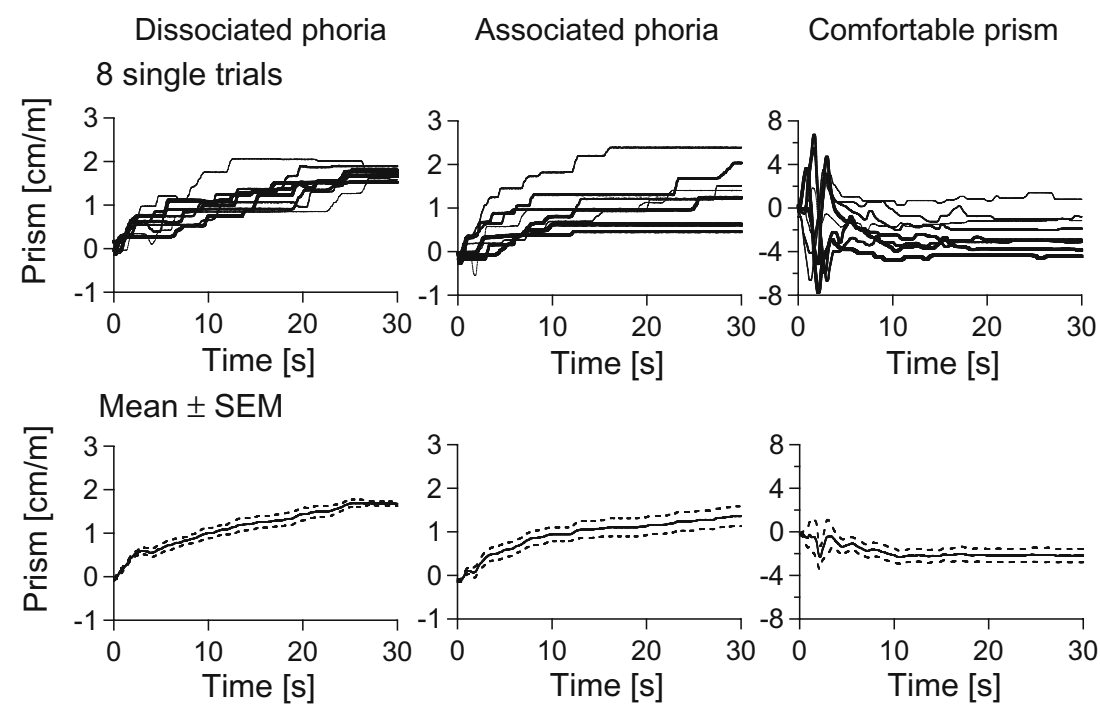


error of the mean) for each observer (Fig. 4). Among the 20 observers, the range of the intertrial mean was for dissociated phoria from +9.3 eso to $-5.9 \mathrm{~cm} / \mathrm{m}$ exo deviation, for associated phoria from +11.2 eso to $-3.3 \mathrm{~cm} / \mathrm{m}$ exo deviation, and for the comfortable prism from +4.8 eso to $-4.1 \mathrm{~cm} / \mathrm{m}$ exo deviation.

To assess the intertrial variability over all 20 observers, we averaged the $95 \%$ confidence interval $( \pm 1.96 \mathrm{SD})$ per session. The values were as follows. Dissociated phoria: first session $\pm 1.3 \mathrm{~cm} / \mathrm{m}$, second session $\pm 1.0 \mathrm{~cm} / \mathrm{m}$; associated phoria: first session $\pm 1.6 \mathrm{~cm} / \mathrm{m}$, second session $\pm 1.6 \mathrm{~cm} / \mathrm{m}$; comfortable prism: first session $\pm 2.0 \mathrm{~cm} / \mathrm{m}$, second session $\pm 2.2 \mathrm{~cm} / \mathrm{m}$. Hence, the variability was similar in the two sessions. Combining the two sessions resulted in a greater overall scatter $(95 \%$ confidence interval $= \pm 1.96 \mathrm{SD}$ ): $\pm 5.8 \mathrm{~cm} / \mathrm{m}$ for dissociated phoria, $\pm 5.4 \mathrm{~cm} / \mathrm{m}$ for associated phoria, and $\pm 4.3 \mathrm{~cm} / \mathrm{m}$ for the comfortable prism. The marked increase in the overall scatter indicates that the values changed between the two sessions. In some cases the mean values of the second session were even outside the $95 \%$ confidence interval of the first session: $5 / 20$ for dissociated phoria, $9 / 20$ for associated phoria, and $6 / 20$ for the comfortable prism. The change was not always concordant in the three conditions. For example, in observer $\# 13$, the associated phoria changed from $+0.1 \pm$ SEM $0.4 \mathrm{~cm} / \mathrm{m}$ to $+4.6 \pm$ SEM $0.5 \mathrm{~cm} / \mathrm{m}$, i.e. in the eso direction, and the comfortable prism changed from $-0.1 \pm$ SEM $0.4 \mathrm{~cm} / \mathrm{m}$ to $-1.8 \pm$ SEM $0.2 \mathrm{~cm} / \mathrm{m}$, i.e. in the exo direction.

Are these changes clinically relevant? As several practitioners consider prescribing prisms from a minimum of about $1.0 \mathrm{~cm} / \mathrm{m}$ onwards [13], we identified the observers in whom the change was greater than $1.0 \mathrm{~cm} / \mathrm{m}$ : for dissociated phoria $6 / 20$ (up to $3.2 \mathrm{~cm} / \mathrm{m}$ ), for associated phoria $11 / 20$ (up to $4.5 \mathrm{~cm} / \mathrm{m}$ ), and for the comfortable prism $12 / 20$ (up to $3.3 \mathrm{~cm} / \mathrm{m}$ ). To find out whether the observers who changed their values by more than $1.0 \mathrm{~cm} / \mathrm{m}$ could be recognized at the first session, we compared the intertrial variability at the first session in two groups: the observers who changed their values more than $1.0 \mathrm{~cm} / \mathrm{m}$ with those who changed them less than $1.0 \mathrm{~cm} / \mathrm{m}$. There was no statistically significant difference between the two groups ( $p=0.67$ for dissociated phoria, 0.11 for associated phoria, 0.52 for the comfortable prism).
Fig. 4 Mean of the last 10 seconds of eight trials \pm SEM for each of the 20 observers. The values obtained in the first session are represented in the left columns, those obtained in the second session in the right columns. $D P=$ dissociated phoria, $A P=$ associated phoria, $C P=$ comfortable prism. The observers are identified with $\# 1$ to $\# 20$. Ordinate: positive values $=$ eso deviation, negative values $=$ exo deviation. Note that the ordinates in \#7 and \#19 are scaled down
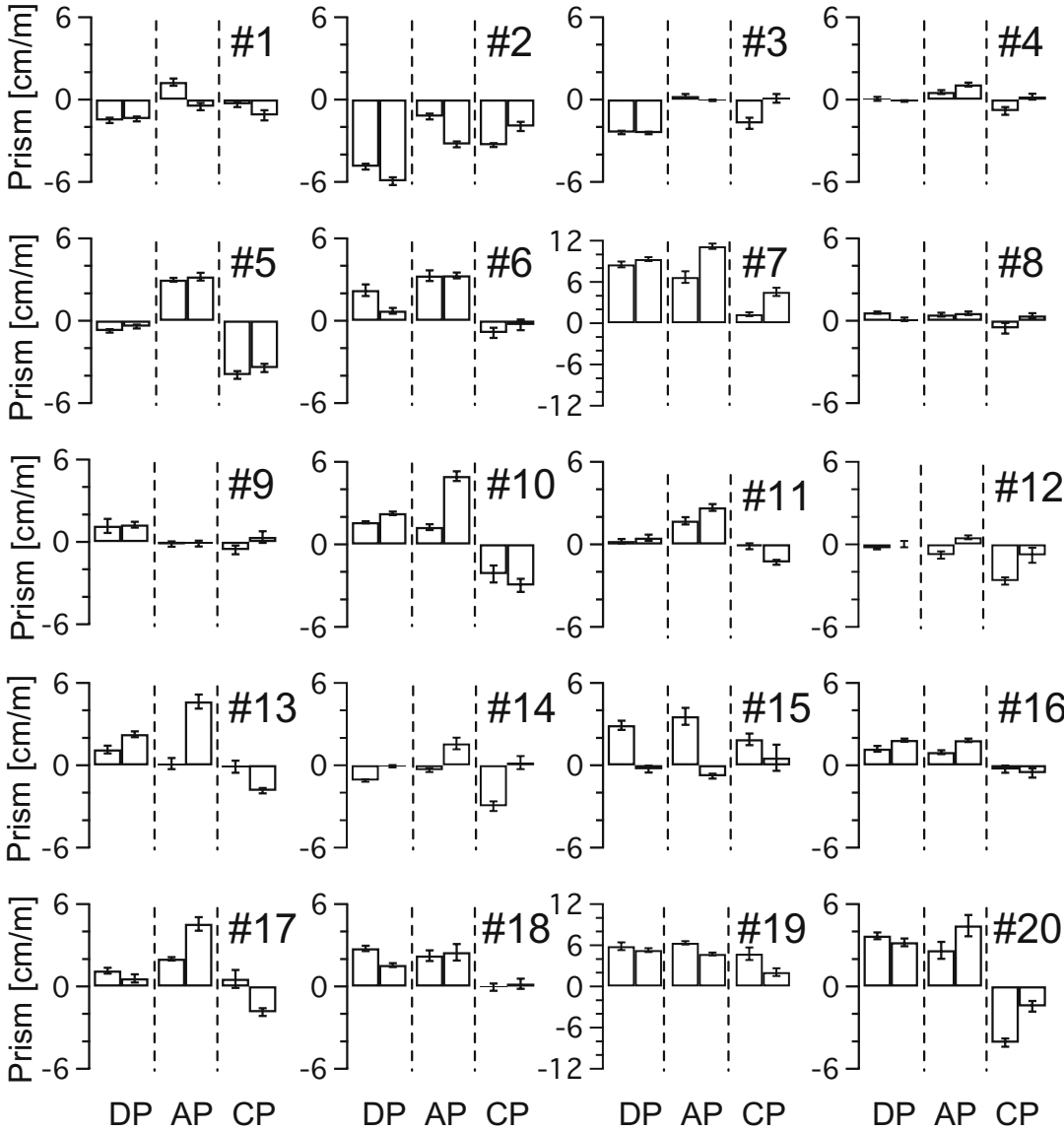
Comparison between dissociated phoria, associated phoria, and the comfortable prism

The ANOVA over all trials of all 20 observers $(n=8 \times 2 \times 20=$ $320)$ revealed that dissociated and associated phoria differed significantly $(p<0.01)$. In 11 observers, the dissociated phoria was lower, in four higher than the associated phoria; in five observers, dissociated and associated phoria were similar.

Figure 5 demonstrates that the majority of observers had an eso deviation for dissociated and associated phoria (13/ 20 and 17/20, respectively), but an exo deviation for the comfortable prism (17/20). The tendency towards eso deviation in the two phoria conditions was also obvious in the average \pm SD over all trials of all 20 observers $(n=8 \times$ $2 \times 20=320):+1.0 \pm 2.9 \mathrm{~cm} / \mathrm{m}$ eso deviation for dissociated phoria, and $+2.0 \pm 2.7 \mathrm{~cm} / \mathrm{m}$ eso deviation for associated phoria, versus $-0.6 \pm 2.2 \mathrm{~cm} / \mathrm{m}$ exo deviation for the comfortable prism. The difference between dissociated phoria and comfortable prism, on the one hand, and the difference between associated phoria and comfortable prism on the other were both significant $(p<0.05$ and $p<0.001$ respectively).

To investigate whether the tendency towards eso deviation in the two phoria conditions, as compared with the comfortable prism, was brought about by the sequence of tests, we made a spot check. We chose observer \#10, because she had a marked eso deviation in the two phoria conditions, but an exo deviation in the comfortable prism. In an extra session, this observer determined the comfortable prism before the tests for dissociated and associated phoria. The values obtained were of the same magnitude as those in the two previous sessions (ANOVA $p=0.084$ for tested difference).

\section{Discussion}

We compared three vergence parameters: dissociated phoria, associated phoria, and the comfortable prism. To avoid any (possibly prejudiced) influence of the experimenter, we recorded the prismatic power set by the observer with a potentiometer. The comfortable prism anticipates the real-life situation in which prismatic spectacles are worn. Therefore, the comfortable prism is very close to the endpoint of therapeutical considerations, i.e. prescribing prismatic spectacles. In contrast, dissociated and associated phoria, determined under artificial viewing conditions, are surrogate measures of the comfortable prism. We studied whether these surrogate measures predict the comfortable prism, assuming that dissociated phoria, associated phoria, and the comfortable prism might be
Fig. 5 Mean of the two sessions \pm SEM for associated phoria, dissociated phoria, and comfortable prism. The first column in each graph represents observer \#1, followed by \#2 etc

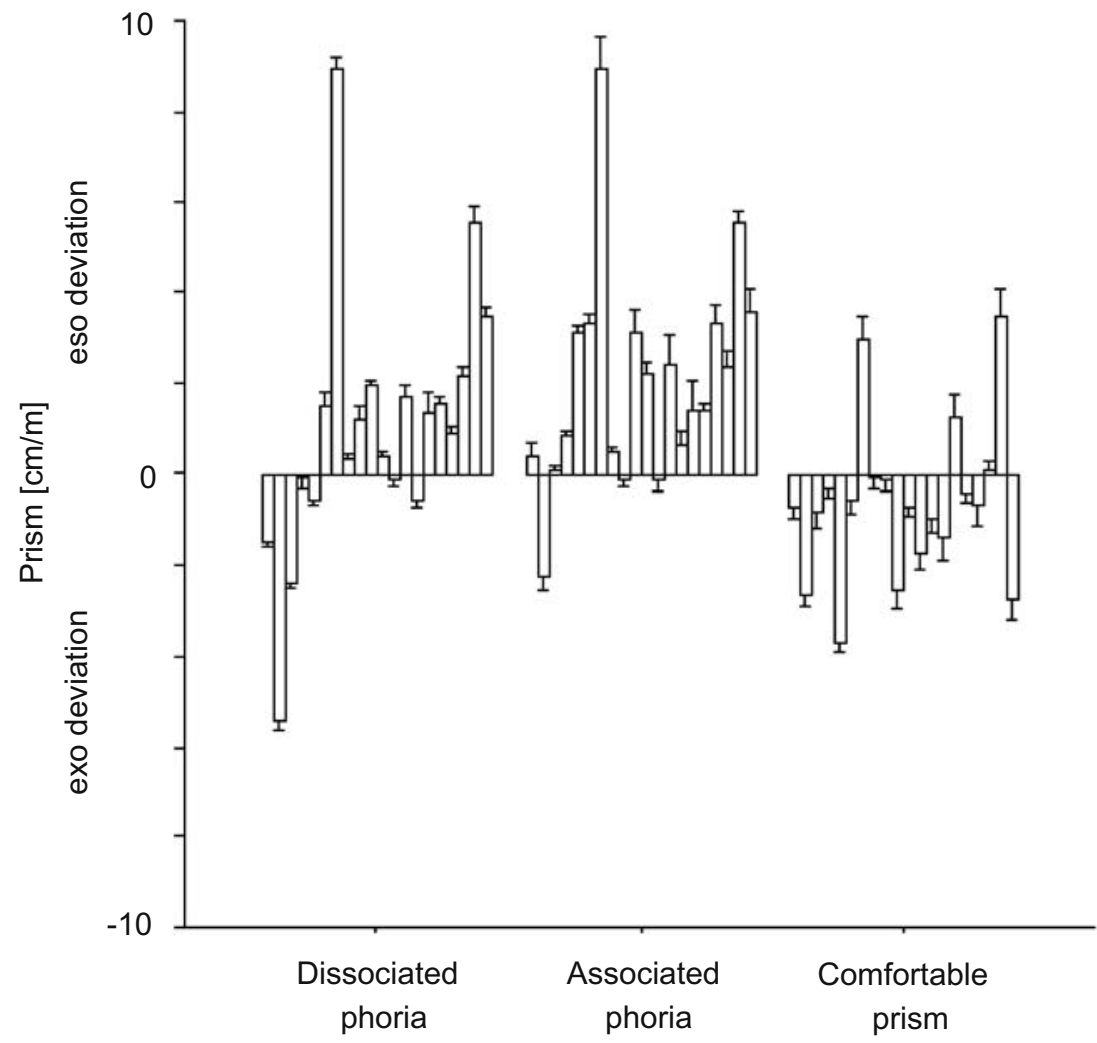


similar, because all three parameters are determined by opening the feedback loop for fusional vergence. When measuring dissociated phoria, fusionable contours are absent; hence, there is no disparity error signal. When associated phoria is measured, any disparity of the (defective) fusional pattern, which initially constitutes an error signal, is promptly nullified by repeated prism adjustment. Concerning the comfortable prism, one has to realize that the fusional feedback loop, which uses disparity as its error signal, can stabilize the vergence angle only if the stimuli are presented to the two eyes in a fixed angle, e.g. through a certain unchanging prism. If, however, the observer is asked to adjust a variable prism according to his or her comfort, the fusional feedback loop is open and cannot stabilize the vergence angle. Instead, the fusional feedback loop is replaced by another feedback loop whose error signal is discomfort. Accordingly, the vergence position reached with the comfortable prism may also be described as the "vergence position of rest when both eyes are exposed to identical pictures".

To our surprise, we found marked differences between the three vergence parameters: as indicated in Fig. 5, the majority of observers had an eso deviation under the two phoria conditions (13/20 for dissociated and 17/20 for associated phoria), but preferred base-in for the comfortable prism, corresponding to an exo deviation (17/20). The maximal difference occurred in observer \#10 who showed in his second session an eso deviation for associated phoria of $+4.9 \mathrm{~cm} / \mathrm{m}$, and an exo deviation for the comfortable prism of $-3.0 \mathrm{~cm} / \mathrm{m}$.

The tendency towards eso deviation in the two phoria conditions was also evident in the significant difference between the averages \pm SD over all 20 observers: $+1.0 \pm$ $2.9 \mathrm{~cm} / \mathrm{m}$ eso deviation for dissociated phoria, $+2.0 \pm 2.7 \mathrm{~cm} /$ $\mathrm{m}$ eso deviation for associated phoria, but $-0.6 \pm 2.2 \mathrm{~cm} / \mathrm{m}$ exo deviation for the comfortable prism. Although the test condition for associated phoria is commonly regarded as being more natural than that for dissociated phoria, the associated phoria was not closer to the comfortable prism than the dissociated phoria. This means that the difference in the overall luminance for the two eyes, present in our test for dissociated phoria, did not push the vergence away from the value obtained with the comfortable prism. This finding supports the finding of Kromeier et al. [7, 8] that unequal luminance of the images for the two eyes does not necessarily influence the vergence position.

What is the reason for the tendency towards eso deviation in the two phoria conditions? A different accommodative demand can be excluded, because we used a similar target in all three conditions. Rather, we suggest that the shift towards eso deviation is brought about by the dissimilarity between the images of the two eyes. This suggestion pertains even to the associated phoria condition, in which ample fusionable contours are available, and the dissimilarity is limited to the monocular Nonius lines. Nevertheless, this dissimilarity was conspicuous for the observers: most of them reported that one or both Nonius lines seemed to disappear every now and then. Correspondingly, the observers reported occasional disappearance of the white dot when they determined their dissociated phoria, although this was less pronounced than the disappearance of the Nonius lines in the associated phoria condition. These observations raise the possibility that the irritating perception of binocular rivalry drives the eso shift in the two phoria conditions. Compatible with this idea is that the eso shift was stronger in the associated than in the dissociated phoria condition, i.e. in the condition with the more pronounced fading.

Considering the open feedback loop for fusional vergence in all three conditions, it is not surprising that the values obtained with all three methods (dissociated phoria, associated phoria, and comfortable prism) were rather variable. This was true in each of the two sessions.

The values changed in many observers from the first to the second session beyond the $95 \%$ confidence interval of the eight trials in the first session. Comparing the three conditions, they did not always change in the same direction (eso or exo). We do not see a specific reason for these changes, as there was no hint that the general health or attentiveness had changed between the two sessions. Rather, the variability might be understandable as there is no need for the ocular motor system to keep the vergence angle stable under open-loop conditions. Surely, adaptation maintains the open-loop vergence in a certain range $[10,12]$, but this range appears to be rather wide.

What are the practical inferences of our study?

As mentioned in the introduction, both dissociated $[11,18]$ and associated phoria [9, 19] have been recommended as indicators for prismatic correction in the case of asthenopia. We purposely did not select our observers according to the presence or absence of asthenopic complaints. Rather, we avoided using the relief from asthenopic complaints as a criterion, because this approach would have required prolonged wearing of various test prisms, including placebo, in a controlled design, to face the following four problems. First, a causal relationship between heterophoria and asthenopia is hard to establish, because the prevalence of both conditions is high in the general population, so that an unrelated coincidence can easily occur. Second, wearing prisms for hours or days leads to adaptation, so that the patient may later value even prisms that he or she initially disliked. Third, most asthenopic symptoms are waxing and waning. It is highly likely that patients seek remedy at a 
time when their symptoms are relatively intense. Hence, a subsequent improvement may well be due to a regression to the mean, rather than to a prism [21]. Fourth, a relief from symptoms may be brought about by the supportive behaviour of the therapist.

Although we did not use the relief from asthenopic complaints as a criterion, we think that our findings relate to the prescription of therapeutic prisms for such patients. The discrepancy between dissociated and associated phoria on the one hand and the comfortable prism on the other hand would probably also occur in patients with asthenopia, and it is plausible to assume that these patients would benefit more from the comfortable prism, chosen by themselves under natural viewing conditions, than from a prescription based on one of the phoria parameters, which carry artefacts due to the artificial test conditions.

Another practical aspect of our study is the finding that both phoria parameters and the comfortable prism can change from one session to the next. Are these changes clinically relevant? Several practitioners consider prescribing prisms from $1.0 \mathrm{~cm} / \mathrm{m}$ onwards [13]. On this background, our finding that 12 of the 20 observers changed their comfortable prism by more than $1.0 \mathrm{~cm} / \mathrm{m}$ (up to $3.3 \mathrm{~cm} / \mathrm{m}$ ) within an interval of a few weeks is important. We examined whether patients tending to a long-term change of their open-loop vergence might be identifiable in the first session by a large scatter. Unfortunately, our results did not support this notion: the intertrial variability at the first session was not larger in the observers who changed their values more than $1.0 \mathrm{~cm} / \mathrm{m}$, as compared with observers who changed their values less than $1.0 \mathrm{~cm} / \mathrm{m}$. Hence, repeated determinations of the comfortable prism on different days appear advisable to identify patients in whom the values remain relatively stable. Only in these patients would a prescription of prisms be reasonable.

What are the limitations of our study?

Towards the end of our experiments, we considered that it might have been preferable to randomize the sequence of tests between the 20 observers. However, it is unlikely that such a randomization would have produced significantly different results, for the following two reasons: (1) an influence of training or fatigue is unlikely since there was no trend between the eight trials of each test, e.g. with the early trials yielding smaller values than the late ones, or vice versa $(p=0.34)$, and (2) a spot check in observer \#10 with the reverse order of tests confirmed this observer's marked discrepancy between an eso deviation in the two phoria conditions, and an exo deviation in the comfortable prism (ANOVA $p=0.084$ for tested difference).

The angles encountered in our 20 observers were relatively small: the dissociated phoria ranged from
$+9.3 \mathrm{~cm} / \mathrm{m}$ eso to $-5.9 \mathrm{~cm} / \mathrm{m}$ exo deviation, the associated phoria from $+11.2 \mathrm{~cm} / \mathrm{m}$ eso to $-3.3 \mathrm{~cm} / \mathrm{m}$ exo deviation, and the comfortable prism from $+4.8 \mathrm{~cm} / \mathrm{m}$ eso to $-4.1 \mathrm{~cm} / \mathrm{m}$ exo deviation. An extrapolation of our conclusions to observers with larger angles may not be justified.

Our tests corresponded to just one of many "natural" viewing conditions: with respect to luminance, visual angle, and distance ( 4 meters), the conditions were similar to watching television. According to previous work [2,3], it is likely that, at closer viewing distances, the values would be shifted in the exo direction. Hence, we suggest that the practitioner should determine the comfortable prism in the viewing distance, for which therapeutic prisms are being considered. This recommendation corresponds with the concept of Jaschinski [4], who suggested that observers should choose their comfortable viewing distance for computer screens in the real-life situation.

Acknowledgement $\mathrm{We}$ are grateful to the Ernst und Berta Grimmke-Stiftung for supporting this research.

\section{References}

1. Evans JW (2002) Pickwell's binocular vision anomalies: investigation and treatment. Butterworth-Heinemann, Oxford

2. Francis EL, Owens DA (1983) The accuracy of binocular vergence for peripheral stimuli. Vision Res 23:13-19

3. Jaschinski W (2001) Fixation disparity and accommodation for stimuli closer and more distant than oculomotor tonic positions. Vision Res 41:923-933

4. Jaschinski W (2002) The proximity-fixation-disparity curve and the preferred viewing distance at a visual display as an indicator of near vision fatigue. Optom Vis Sci 79:158-169

5. Jenkins TC, Pickwell LD, Yekta AA (1989) Criteria for decompensation in binocular vision. Ophthalmic Physiol Opt 9:121-125

6. Karania R, Evans BJ (2006) The Mallett Fixation Disparity Test: influence of test instructions and relationship with symptoms. Ophthalmic Physiol Opt 26:507-522

7. Kromeier M, Schmitt C, Bach M, Kommerell G (2001) Heterophoria measured with white, dark-grey and dark-red Maddox rods. Graefes Arch Clin Exp Ophthalmol 239:937-940

8. Kromeier M, Schmitt C, Bach M, Kommerell G (2002) Vergleich zwischen dissoziierter und assoziierter Heterophorie [Comparison between dissociated and associated heterophoria]. Ophthalmologe 99:549-554

9. Mallett RFJ (1966) A fixation disparity test for distance use. The Optician 152:1-9

10. Miles FA, Judge SJ, Optican LM (1987) Optically induced changes in the couplings between vergence and accommodation. J Neurosci 7:2576-2589

11. van Noorden GK, Campos EC (2002) Binocular vision and ocular motility: theory and management of strabismus. Mosby, St. Louis

12. North RV, Sethi B, Henson DB (1986) Effects of prolonged forced vergence upon the adaptation system. Ophthalmic Physiol Opt 6:391-396

13. O’Leary CI, Evans BJ (2003) Criteria for prescribing optometric interventions: literature review and practitioner survey. Ophthalmic Physiol Opt 23:429-439 
14. Ogle KN, Mussey F, Prangen AD (1949) Fixation disparity and the fusional processes in binocular single vision. Am J Ophthalmol 32:1069-1087

15. Palmer EA, van Noorden GK (1978) The relationship between fixation disparity and heterophoria. Am J Ophthalmol 86:172-176

16. Pickwell LD, Kaye NA, Jenkins TC (1991) Distance and near readings of associated heterophoria taken on 500 patients. Ophthalmic Physiol Opt 11:291-296

17. Risley (1889) A new rotary prism. Trans Amer Ophthalmol Soc 5:412
18. Sheard C (1930) Zones of ocular comfort. American Journal of Optometry 7:9-25

19. Sheedy JE, Saladin JJ (1978) Association of symptoms with measures of oculomotor deficiencies. Am J Optom Physiol Opt 55:670-676

20. Yekta AA, Pickwell LD, Jenkins TC (1989) Binocular vision, age and symptoms. Ophthalmic Physiol Opt 9:115-120

21. Yudkin PL, Stratton IM (1996) How to deal with regression to the mean in intervention studies. Lancet 347:241-243 